\title{
Arthrosen
}

\section{Konservative Behandlungsmöglichkeiten nutzen}

_ Bei der Behandlung der Arthrose nehmen konservative Maßnahmen wie Bewegungs- und nutritive Therapien einen immer größeren Stellenwert ein, wie Dr. med. Frank Weinert, niedergelassener Allgemein- und Sportmediziner in Gangkofen, darlegte. Gerade die Kombination von Krafttraining und Zufuhr multipler Nährstoffe hat mittlerweile gut belegte synergistische Effekte.

Jüngst wurde auch die sehr gute symptomatische Wirkung der ergänzenden bilanzierten Diät Orthomol arthroplus bei Gonarthrose in einer kontrollierten Studie belegt, die Weinert vorstellte [Heisel J et al. Orthop Unfallchir Praxis. 2015;4(7-8):388-94]. Eine Metaanalyse ermittelte außerdem den Einfluss verschiedener Trainingsarten auf den Schmerz bei Knie- und Hüftgelenksarthrose. Dabei erzielte Krafttraining die größten Effektstärken (rund 0,8), gefolgt von Flexibilitäts- und Ausdauertraining [Uthman OA et al. BMJ. 2013;374:f5555].

\section{Bewegung plus Ernährung lindert Knieschmerz}

Training plus gute Ernährung, in diesem Fall die Supplementierung von Omega-3-reichem Fischöl hatte signifikant mehr Trainingseffekte im Sinne eines Muskelaufbaus, als Krafttraining allein [Rodacki CL et al. Am J Clin Nutr. 2012;95: 428-36]. Ob die ergänzende bilanzierte Diät Orthomol arthroplus ${ }^{\circ}$ belegbare Effekte hat, sollte eine multizentrische, placebokontrollierte Doppelblindstudie ermitteln, in die $366 \mathrm{~Pa}$ tienten mit Gonarthrose eingeschlossen wurden. Sie erhielten täglich über drei Monate entweder Orthomol arthroplus ${ }^{\circledR}$ oder Placebo.

Die Responder-Rate betrug in der Verumgruppe 52,5\% der Patienten vs. 36,8\% im Placebokollektiv (Intention-to-treatKollektiv; $p=0,003$ ). Als gebessert oder sehr gebessert beurteilten die Ärzte die Befunde bei $71,9 \%$ der Patienten der Verumgruppe (vs. 53,6\% unter Placebo,

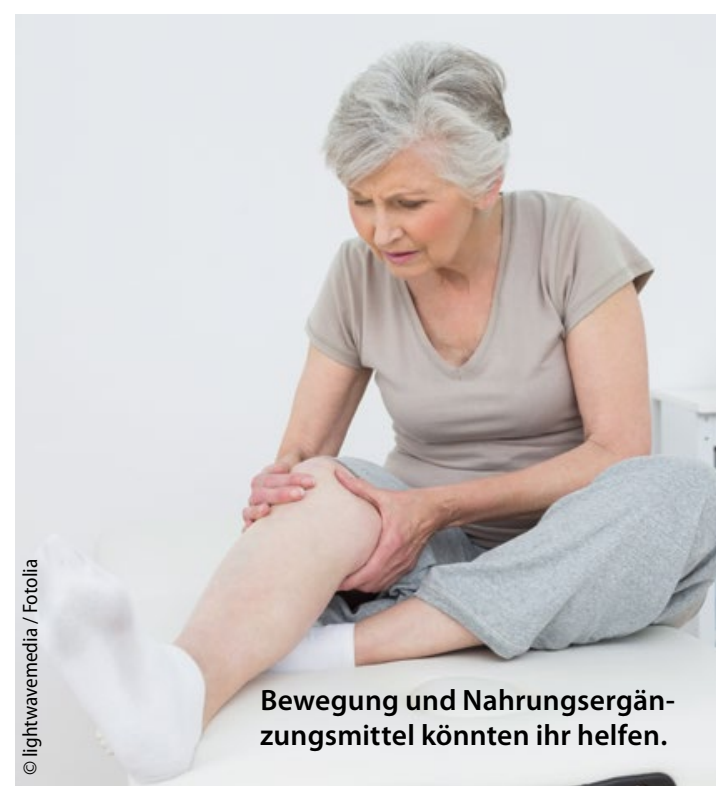

$\mathrm{p}<0,01)$ [Heisel J et al. Orthop Unfallchir Praxis. 2015;4(7-8):388-94].

Reimund Freye

- Pressegespräch „Ernährungsmedizin im Rahmen der Arthrosebehandlung", 64. Jahrestagung der Vereinigung Süddeutscher Orthopäden und Unfallchirurgen; Baden-Baden April 2016; (Veranstalter: Orthomol)

\section{Neue Servicematerialien}

\section{Hochdruck und Schmerz - einfach verstehen}

_ Im Praxis- und Apothekenalltag beraten Ärzte und Apotheker immer häufiger Patienten mit geringen Deutschkenntnissen [https://www.bundesregierung.de/Content/DE/_Anlagen/IB/ 2014- 10-29-Lagebericht-lang.pdf? $\mathrm{blob}=$ publicationFile\&v $=4$. Letzter Aufruf: 06.04.2016.]. Denn mittlerweile leben in Deutschland über 16 Millionen Menschen mit Migrationshintergrund [https://www.destatis.de/DE/ZahlenFakten/GesellschaftStaat/Bevoelkerung/ MigrationIntegration/Migrationshintergrund/Tabellen.html. Letzter Aufruf: 06.04.2016].
Sprachbarrieren führen jedoch oftmals dazu, dass die Aufklärung von Patienten zeitintensiver und aufwändiger wird. Mit kostenlosen Abgabematerialien für die Indikationsgebiete Hochdruck und Schmerz in den Sprachen Englisch, Französisch, Türkisch und Arabisch unterstützt 1 A Pharma nun Ärzte und Apotheker bei der Patientenberatung. Folgende Materialien stehen zur Verfügung:

- Ratgeber Schmerz zur Information über Schmerzentstehung, verschiedene Schmerzarten und Behandlungsmöglichkeiten,
- Schmerztagebuch und Schmerzskala zur besseren Dokumentation von Schmerzen,

- Ratgeber Bluthochdruck zur Information über Ursachen und verschiedene Behandlungsmöglichkeiten und ein

- Blutdruckpass für die regelmäßige Dokumentation des Blutdrucks.

Ärzte und Apotheker können die Abgabematerialien ab sofort kostenlos per Fax unter 0800 / 6131111 oder unter www.1apharma.de bestellen.

\section{Red.}

